\title{
Structure and dynamics of the class I young stellar object L1489 IRS
}

\author{
C. Brinch ${ }^{1}$, A. Crapsi ${ }^{1}$, M. R. Hogerheijde ${ }^{1}$, and J. K. Jørgensen ${ }^{2}$ \\ ${ }^{1}$ Leiden Observatory, Leiden University, PO Box 9513, 2300 RA Leiden, The Netherlands \\ e-mail: brinch@strw. leidenuniv.nl \\ 2 Harvard-Smithsonian Center for Astrophysics, 60 Garden Street, Mail Stop 42, Cambridge, MA 02138, USA
}

Received 21 April 2006 / Accepted 11 October 2006

\begin{abstract}
Context. During protostellar collapse, conservation of angular momentum leads to the formation of an accretion disc. Little is known observationally about how and when the velocity field around the protostar shifts from infall-dominated to rotation-dominated. Aims. We investigate this transition in the low-mass protostar L1489 IRS, which is known to be embedded in a flattened, disc-like structure that shows both infall and rotation. We aim to accurately characterise the structure and composition of the envelope and its velocity field, and find clues to its nature.

Methods. We construct a model for L1489 IRS consisting of an flattened envelope and a velocity field that can vary from pure infall to pure rotation. We obtain best-fit parameters by comparison to 24 molecular transitions from the literature, and using a molecular excitation code and a Voronoi optimisation algorithm. We test the model against existing millimeter interferometric observations, near-infrared scattered light imaging, and ${ }^{12} \mathrm{CO}$ ro-vibrational lines.

Results. We find that L1489 IRS is well described by a central stellar mass of $1.3 \pm 0.4 M_{\odot}$ surrounded by a $0.10 M_{\odot}$ flattened envelope with approximate scale height $h \approx 0.57 R$, inclined at $74_{-17^{\circ}}^{\circ}$. The velocity field is strongly dominated by rotation, with the velocity vector making an angle of $15^{\circ} \pm 6^{\circ}$ with the azimuthal direction. Reproducing low-excitation transitions requires that the emission and absorption by the starless core 1' (8400 AU) east of L1489 IRS is included properly, implying that L1489 IRS is located partially behind this core.

Conclusions. We speculate that L1489 IRS was originally formed closer to the center of this core, but has migrated to its current position over the past few times $10^{5} \mathrm{yr}$, consistent with their radial velocity difference of $0.4 \mathrm{~km} \mathrm{~s}^{-1}$. This suggests that L1489 IRS' unusual appearance may be result of its migration, and that it would appear as a "normal" embedded protostar if it were still surrounded by an extended cloud core. Conversely, we hypothesize that the inner envelopes of embedded protostars resemble the rotating structure seen around L1489 IRS.
\end{abstract}

Key words. ISM: kinematics and dynamics - ISM: molecules - ISM: individual objects: L1489 IRS - radio lines: ISM stars: formation

\section{Introduction}

The last three decades has provided a detailed understanding of the process of low-mass star formation through theoretical work and advancements in the observational facilities (see for example the review by André et al. 2000; or several reviews in Reipurth et al. 2006). These achievements have given us a detailed view on infant stars through their various stages of formation. Lowmass stars form out of dark molecular clouds when dense regions can collapse under the influence of their own gravity. When sufficient density is reached, a protostellar object is formed, still deeply embedded in a surrounding envelope. Conservation of angular momentum leads to the formation of a disc around the protostar onto which the surrounding dust and gas is accreted, although little details are known of how exactly discs grow. As the stellar wind starts to clear out the envelope, the star and the disc becomes visible in the optical and infrared and the object enters the classical T Tauri stage which then later evolves into a main sequence star (Shu 1977; Lizano \& Shu 1989; Adams et al. 1988). Most observed Young Stellar Objects (YSOs) are usually classified based on the shape of their spectral energy distribution (SED) as either a Class I, II, or III (Lada \& Wilking 1984). Class I objects are deeply embedded in dense cores, while Class II objects are surrounded by actively accreting discs. Class III objects have little material left in a disc, but are still descending to the Main Sequence. Sometimes, however, a YSO does not clearly fit into one of these categories. Those objects are most likely the ones that can shed light on some of the missing pieces of the picture. In this paper we study one such transitional object, L1489 IRS, and investigate the structure, dynamics, and composition of its circumstellar material.

L1489 IRS (IRAS 04016+2610) is classified as a Class I object based on its SED and visibility at near-infrared wavelengths (Myers et al. 1987). Like many embedded YSOs, line profiles of dense gas tracers like $\mathrm{HCO}^{+} J=3-2$ and 4-3 show red-shifted absorption dips usually interpreted as indications of inward motions in the envelopes (Gregersen \& Evans 2000; Mardones et al. 1997). However, Hogerheijde (2001) shows that the spatially resolved $\mathrm{HCO}^{+} J=1-0$ emission exhibits a flattened, $2000 \mathrm{AU}$ radius structure dominated by Keplerian rotation. In this aspect, L1489 IRS more closely resembles a T Tauri star with a circumstellar disc (Koerner \& Sargent 1995; Guilloteau \& Dutrey 1998; Simon et al. 2000). T Tauri discs, however, are in general much smaller than the disc structure seen in L1489 IRS with radii of several hundreds of AU. Scattered light imaging by Padgett et al. (1999) shows the central stellar object 
and the presence of a slightly flaring dark lane, consistent with the disc-like configuration inferred from the $\mathrm{HCO}^{+} 1-0$ observations. Careful analysis if the circumstellar velocity field by Hogerheijde (2001) revealed that infalling motions are present at $\sim 10 \%$ of the Keplerian velocities. Hogerheijde hypothesized that L1489 IRS is in a short-lived transitional stage between a collapsing envelope (Class I) and a viscously supported, Keplerian disc (Class II). Observations of ro-vibrational $\mathrm{CO}$ absorption lines at $4.7 \mu \mathrm{m}$ showed that the inward motions continue to within 1 AU from the central star (Boogert et al. 2002).

In this paper we address a number of questions about L1489 IRS. We construct a model for the circumstellar structure that accommodates all observations, ranging from an extensive set of single-dish molecular line measurements to the interferometric observations, the scattered light imaging, and the CO ro-vibrational absorption lines. Hogerheijde (2001) adopted a flared disc model with a fixed scale height for the structure inspired by the interferometric imaging. In this Paper we choose a description for the circumstellar structure that can be smoothly varied from spherical to highly flattened, and investigate if the full data set requires a disc-like configuration. We also adopt a velocity field that can range from purely Keplerian to completely free-fall, or any combination of the two. By considering the full data set, stronger constraints can be set on the velocity field and the dynamical state of L1489 IRS than possible before. We perform a rigorous optimisation of the model for L1489 IRS using all available single-dish line data, and test the model by comparing the interferometric observations, the scattered light imaging, and the $\mathrm{CO}$ ro-vibrational absorption lines to predictions from the model. Once we have established a satisfactory model, also taking into account the immediate cloud environment, we explore the nature of L1489 IRS. Does it represent a transitional state between Class I and II? Do all YSOs go through this stage? Or is L1489 IRS is some way special?

The layout of this Paper is as follows. Section 2 present our data set and a detailed overview of the model and fit optimisation procedure. Section 3 describes our best fit model and its reliability, including comparison with observations of interferometric observations, scattered light imaging, and CO ro-vibrational absorption lines. Section 4 discusses our results in the light of the nature and evolutionary state of L489 IRS and explores the wider implications for our understanding of star formation. Section 5 concludes the Paper with a brief summary.

\section{Observations and model description}

\subsection{Single-dish observations}

The primary data set on L1489 IRS used in this paper was published by Hogerheijde et al. (1997) and Jørgensen et al. (2004), and consists of 24 transitions among 12 molecular species. Figure 1 shows all 24 spectra. Table 1 lists the transitions, integrated line strengths, line widths, and relevant beam sizes of the single-dish telescopes. In all cases, line intensities are on the main-beam antenna temperature scale, using the appropriate beam efficiencies. The integrated intensities are obtained by fitting a Gaussian to the line. In some cases, no lines are visible above the noise level, and $3 \sigma$ upper limits are given. The signalto-noise ratio of the $\mathrm{HNC} 4-3$ and $\mathrm{H}_{2} \mathrm{CO} 5_{15}-4_{14}$ spectra was insufficient for a proper Gaussian fit; instead the spectra are simply integrated from -4 to $+4 \mathrm{~km} \mathrm{~s}^{-1}$ with respect to the systemic velocity of $+7.2 \mathrm{~km} \mathrm{~s}^{-1}$. In addition to these molecular line data, we also use the total mass derived from the $850 \mu \mathrm{m}$ continuum observations by JCMT/SCUBA (Hogerheijde \& Sandell 2000).
Apart from the single dish data which we use in the model optimisation, we compare predictions by the model to other previously published observations: a $\mathrm{HCO}^{+} J=1-0$ interferometer map from the BIMA and OVRO arrays (Hogerheijde et al. 1998), CO ro-vibrational absorption spectra from the Keck Telescope (Boogert et al. 2002), and the near-infrared scattered light imaging from HST/NICMOS (Padgett et al. 1999).

\subsection{The model}

Previous studies of L1489 IRS clearly indicate that an axisymmetric description of its circumstellar structure is required. In the following subsections we construct a description of the density $n(r, \theta)$, gas temperature $T(r, \theta)$, and velocity field $\boldsymbol{v}(r, \theta)=$ $\left(v_{R}, v_{z}, v_{\phi}\right)$. Throughout we attempt to keep the number of free parameters at a minimum. In the end we arrive at four free parameters, in addition to the eight molecular abundances which we fit but assume constant throughout the source, and seven parameters that we hold fixed (Table 2).

\subsubsection{Density}

We adopt an axi-symmetric description of the gas density $n(R, z)$ consistent with the spherical model from (Jørgensen et al. 2002). These authors deduce a total mass of $0.097 M_{\odot}$ and a density following a radial power Law with slope -1.8 between radii of 7.8 and $9360 \mathrm{AU}$. We truncate this model at the observed outer radius of L1489 IRS of 2000 AU, but keep the power-law slope and mass conserved. Instead of a simple radial power law, $n \propto r^{-p}$ with $p=1.8$, we adopt a Plummer-like profile, $n \propto\left[1+\left(r / r_{0}\right)^{2}\right]^{-p / 2}$ with $r_{0}=4.0$ AU. This description keeps the density finite at all radii, but since $r_{0}$ is much smaller than the scales of interest here, the resulting density distribution is identical to that used by Jørgensen et al. (2002).

From this spherically symmetric density distribution we construct an axi-symmetric, flattened configuration by multiplying by a factor $\sin ^{f} \theta$, where $f$ can take any value $\geq 0$ (see Stamatellos et al. 2004, where this approach was used for modelling starless cores). The adopted density distribution now becomes,

$n(r, \theta)=n_{0}\left(1+\left(\frac{r}{r_{0}}\right)^{2}\right)^{-p / 2} \sin ^{f} \theta$.

For $f=0$ this reduces to a spherically symmetric structure, while for $f>10$ the resulting profiles becomes largely indistinguishable as they approach a step function (Fig. 2). The mass contained in the structure is kept constant at $0.097 M_{\odot}$ by adjusting $n_{0}$ as $f$ is varied. The only free parameter in the density description is the flattening parameter $f$.

\subsubsection{Temperature}

The temperature of the gas and the dust (which we assume to be identical) in the circumstellar structure of L1489 IRS depends on the stellar luminosity which is $\sim 3.7 L_{\odot}$ (Kenyon et al. 1993a) and the infrared radiative transfer through the structure. Since most of the circumstellar material is optically thin to farinfrared radiation, the deviations introduced by the flattening on the temperature structure are minor. Furthermore, the line excitation does not depend strongly on small temperature differences. A spherically symmetric description of the temperature therefore suffices. Using the continuum radiation transfer code DUSTY (Nenkova et al. 1999) and the density structure of Eq. (1) with 


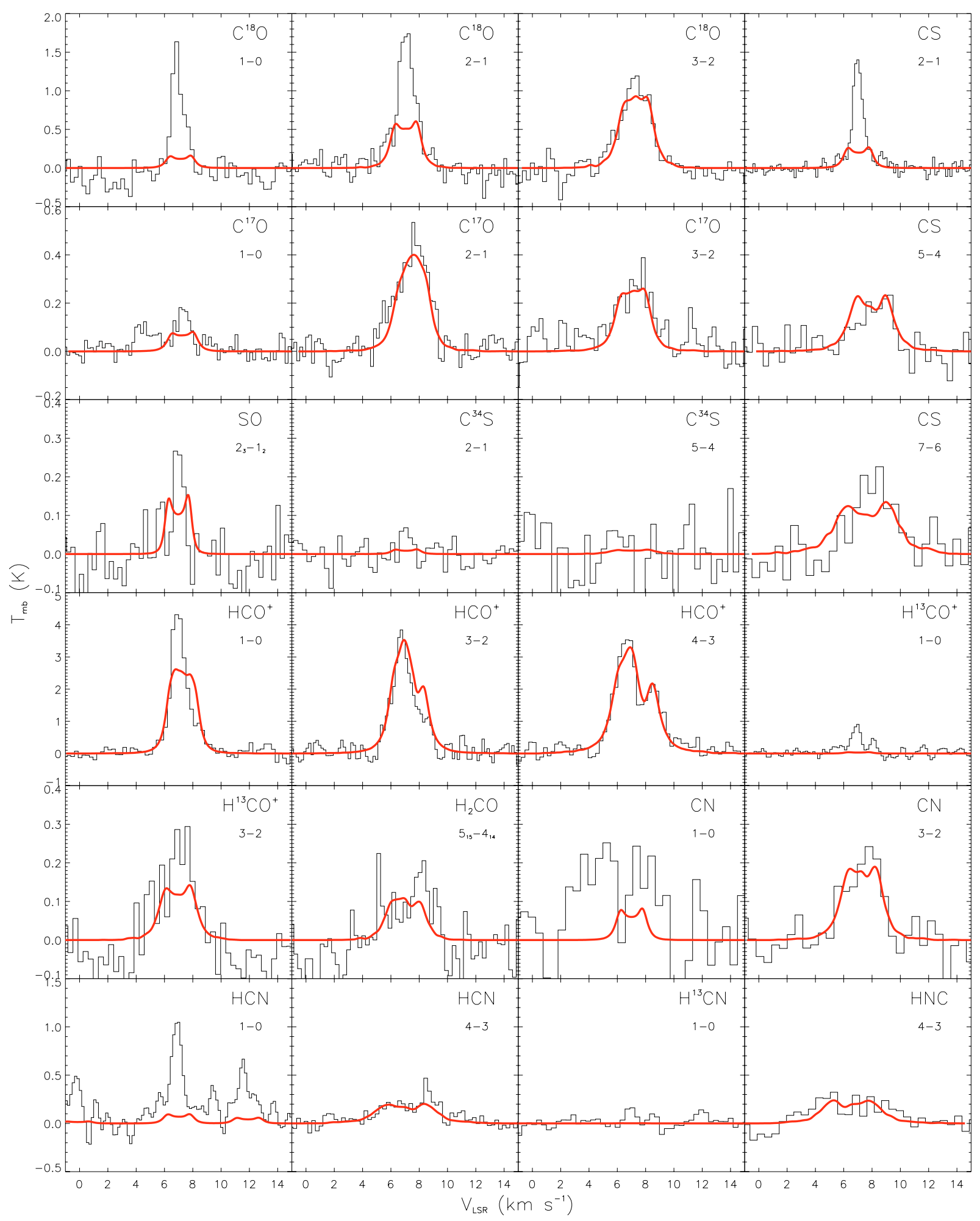

Fig. 1. The 24 single-dish molecular line spectra used for the model optimisation (histograms). The solid lines show the best-fit results for the model of L1489 IRS (see also Fig. 5).

$p=1.8$ and $f=0$, we find that the temperature is well described by,

$T(r)=19.42 \mathrm{~K}\left(\frac{r}{1000 \mathrm{AU}}\right)^{-0.35}$.

There are no free parameters in this description of the temperature.

\subsubsection{Velocity field}

The velocity field is parameterized by a central, stellar mass $M_{\star}$ and an angle $\alpha$ in such a way that,

$v_{r}=-\sqrt{2} \sqrt{\frac{G M_{\star}}{r}} \sin \alpha$, 
Table 1. Single-dish molecular line data set.

\begin{tabular}{|c|c|c|c|c|}
\hline Molecule & Transition & $\underset{\left(\mathrm{K} \mathrm{km} \mathrm{s}{ }^{-1}\right)}{T_{\mathrm{mb}} \mathrm{d} v}$ & $\begin{array}{c}F W H M \\
\left(\mathrm{~km} \mathrm{~s}^{-1}\right) \\
\end{array}$ & $\begin{array}{c}\text { Beam } \\
\left({ }^{\prime \prime}\right)\end{array}$ \\
\hline \multirow[t]{3}{*}{$\mathrm{C}^{18} \mathrm{O}$} & $1-0$ & $1.8 \pm 0.04$ & 1.1 & 34 \\
\hline & $2-1$ & $2.8 \pm 0.1$ & 1.6 & 23 \\
\hline & $3-2$ & $3.4 \pm 0.1$ & 2.5 & 15 \\
\hline \multirow[t]{3}{*}{$\mathrm{C}^{17} \mathrm{O}$} & $1-0$ & $0.5^{a} \pm 0.04$ & 2.9 & 22 \\
\hline & $2-1$ & $1.2^{a} \pm 0.03$ & 2.6 & 11 \\
\hline & $3-2$ & $0.7 \pm 0.1$ & 2.7 & 15 \\
\hline \multirow[t]{3}{*}{ CS } & $2-1$ & $1.2 \pm 0.02$ & 0.9 & 38 \\
\hline & $5-4$ & $0.6 \pm 0.04$ & 0.8 & 22 \\
\hline & $7-6$ & $0.7 \pm 0.1$ & 3.6 & 15 \\
\hline \multirow[t]{2}{*}{$\mathrm{C}^{34} \mathrm{~S}$} & $2-1$ & $<0.3^{b}$ & - & 39 \\
\hline & $5-4$ & $<0.3^{b}$ & - & 21 \\
\hline \multirow{3}{*}{$\mathrm{HCO}^{+}$} & $1-0$ & $6.7 \pm 0.2$ & 2.1 & 28 \\
\hline & $3-2$ & $6.9 \pm 0.2$ & 2.2 & 19 \\
\hline & $4-3$ & $10.0 \pm 0.3$ & 2.5 & 14 \\
\hline \multirow[t]{2}{*}{$\mathrm{H}^{13} \mathrm{CO}^{+}$} & $1-0$ & $0.8 \pm 0.1$ & 0.8 & 43 \\
\hline & $3-2$ & $0.8 \pm 0.1$ & 1.8 & 19 \\
\hline \multirow[t]{2}{*}{$\mathrm{HCN}$} & $1-0$ & $2.4^{a} \pm 0.1$ & 2.3 & 43 \\
\hline & $4-3$ & $1.3 \pm 0.1$ & 5.8 & 14 \\
\hline $\mathrm{H}^{13} \mathrm{CN}$ & $1-0$ & $<0.5^{b}$ & - & 44 \\
\hline \multirow[t]{2}{*}{$\mathrm{CN}$} & $1_{023}-0_{012}$ & $0.63 \pm 0.12$ & - & 33 \\
\hline & $3-2$ & $0.67^{a} \pm 0.09$ & 3.9 & 15 \\
\hline $\mathrm{HNC}$ & $4-3$ & $1.5 \pm 0.3$ & 6.4 & 14 \\
\hline $\mathrm{H}_{2} \mathrm{CO}$ & $5_{15}-4_{14}$ & $0.61 \pm 0.07$ & 4.0 & 14 \\
\hline SO & $2_{3}-1_{2}$ & $0.3 \pm 0.03$ & 0.8 & 38 \\
\hline
\end{tabular}

${ }^{a}$ Intensity integrated over multiple hyperfine components. ${ }^{b}$ No line detected. $3 \sigma$ upper limit, based on an assumed line width of $1.5 \mathrm{~km} \mathrm{~s}^{-1}$. The error bars on the intensity does not include the $20 \%$ calibration error.

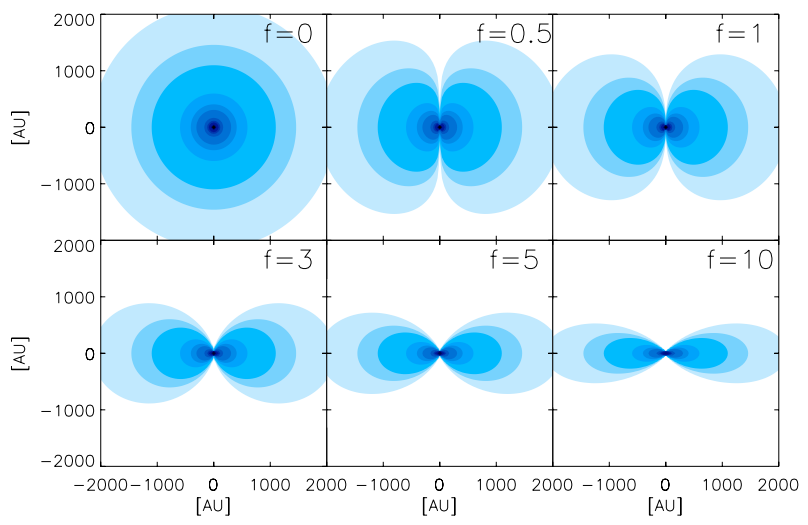

Fig. 2. Progression of flattening of the adopted density structure as $f$ is increased from 0 (purely spherical) to 10 in Eq. (1).

$v_{\phi}=\sqrt{\frac{G M_{\star}}{r}} \cos \alpha$.

For $\alpha=0$ this reduces to pure Keplerian rotation around a mass $M_{\star}$ without any inward motions; for $\alpha=\frac{\pi}{2}$ the velocity field is that of free fall to a mass $M_{\star}$. Intermediate values of $\alpha$ produce a velocity field where material spirals inward. The implicit assumption in this description is that both components of the velocity field vary inversely proportional with $\sqrt{r}$. Note that $\alpha$ should not be confused with the geometric angle determining the direction of the flow lines.

In this description there are two free parameters, the stellar mass $M_{\star}$ and the angle $\alpha$ which is kept constant with radius. In addition to this ordered velocity field, we add a turbulent velocity field with FWHM $0.2 \mathrm{~km} \mathrm{~s}^{-1}$.

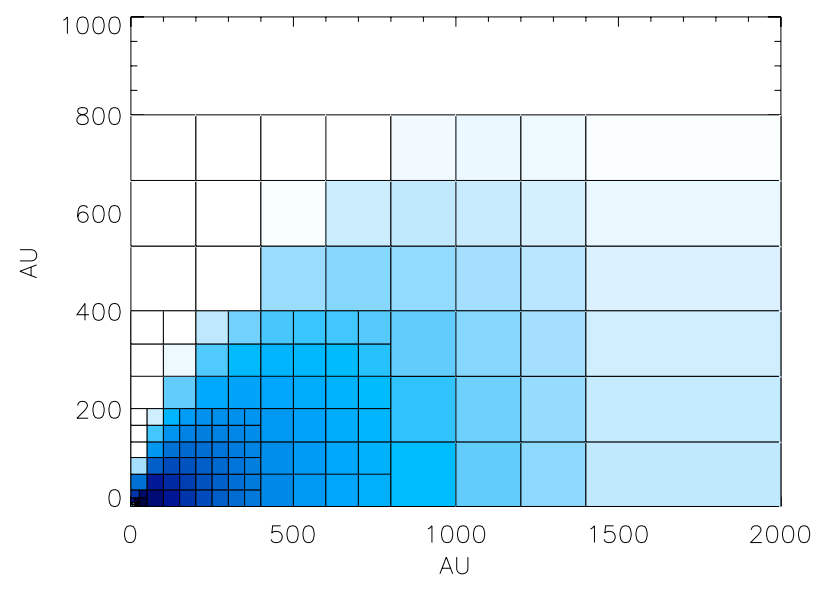

Fig. 3. Layout of the grid cells for a model with $f=3.8$.

\subsection{Molecular excitation and line radiative transfer}

The excitation of the molecules and the line radiative transfer is calculated using the Accelerated Monte Carlo code RATRAN (Hogerheijde \& van der Tak 2000). Collisional excitation rates are taken from the Leiden Atomic and Molecular Database LAMDA (Schöier et al. 2005). We lay out the model onto three nested $8 \times 6$ grids (Fig. 3). The innermost grid cell is subdivided four times, so that the innermost cell is resolved down to $4 \mathrm{AU}$. All properties are calculated as cell averages, by numerically integration over the cell and divide by its volume. To reduce computing time, cells with $\mathrm{H}_{2}$ densities below $10^{3} \mathrm{~cm}^{-3}$ are dropped. Such cells do not contribute significantly to the line emission or absorption. Dust continuum emission is included through a standard gas-to-dust ratio of 100:1 and dust emissivity from Ossenkopf \& Henning (1994) for thin ice mantles which has been accreted and coagulated for about $10^{5}$ years.

Synthetic observations are created from the molecular excitation by performing ray-tracing after placing the object at a distance of $140 \mathrm{pc}$ and an inclination $i$ (a free parameter). The resulting spectra are convolved with the appropriate Gaussian beams. Figure 1 shows the best-fit model spectra (the best fit is discussed in Sect. 3).

\subsection{Modelling the neighbouring cloud core}

During the optimisation of the fit (Sect. 2.5) it became obvious that several lines, and especially those of lower-lying rotational transitions taken in large beams, were contaminated by emission with small line width. This emission component is especially clear in the $\mathrm{C}^{18} \mathrm{O} 1-0$ and 2-1 lines, the CS 2-1 line, the $\mathrm{HCN}$ 1-0 line, and, to some extent, the $\mathrm{HCO}^{+} 1-0$ line (Fig. 1). The emission has a $V_{\mathrm{LSR}}$ of $6.8 \mathrm{~km} \mathrm{~s}^{-1}$, slightly lower than that of L1489 IRS of $7.2 \mathrm{~km} \mathrm{~s}^{-1}$. Cold fore- or background gas with small turbulent velocity is the likely cause for this component. The $850 \mu \mathrm{m}$ SCUBA map from Hogerheijde \& Sandell (2000) reveals that L1489 IRS sits at the edge of an extended, probably starless, cloud core with a radius of 60" (8400 AU). Cold gas in this core therefore contributes to the low- $J$ emission lines, and especially in spectra taken with large beams.

We construct a simple description for the neighbouring core, so that we can take its emission into account in our optimisation of the model for L1489 IRS, as well as its absorption if this source is located behind the core. We approximate the core as spherical with a radius of $60^{\prime \prime}$, which is roughly the distance of L1489 IRS to its centre. We assume that it is isothermal at $10 \mathrm{~K}$ 
and that it has abundances typical for starless cores (Jørgensen et al. 2004). For the species which does not show any cloud core emission, the abundances are unconstrained and we just set the abundances sufficiently low. In the case of $\mathrm{CO}$ we use an abundance of $5 \times 10^{-5}$. The CS abundance is set to $2 \times 10^{-9}$, and the $\mathrm{HCO}^{+}$and $\mathrm{HCN}$ abundances are $27 \times 10^{-9}$ and $4 \times 10^{-9}$ respectively. We derive its density distribution by fitting the $850 \mu \mathrm{m}$ emission from Hogerheijde \& Sandell (2000). We find an adequate fit for a radial power-law with slope -2 and a density of $4 \times 10^{6} \mathrm{~cm}^{-3}$ at $r=1000$ AU resulting in a cloud mass of $2.9 M_{\odot}$. This is consistent with the drop off in density found in many starless cores on scales $(r>1000 \mathrm{AU})$ that are relevant to us (André et al. 1996). Because it falls outside even our largest beam on L1489 IRS we do not investigate if the density in the neighbouring core levels off at the center, as is seen for many starless cores. The relative smoothness of the $850 \mu \mathrm{m}$ emission suggest that this is the case, however.

Using RATRAN we calculate the expected emission and the optical depth of each of the observed transitions. In our model optimisation procedure (see below), the emission from L1489 IRS and the neighbouring core are added on a channelby-channel basis, with the appropriate spatial offset for the core. We find that we can only make a fit that is reasonable if L1489 IRS is located behind the core; we need both the emission and the opacity of the cloud. This is taken into account by first attenuating the emission from L1489 IRS by the core's opacity, again on a channel-by-channel basis, and subsequently adding the core's emission in each channel, followed by beam convolution.

In this section we derived only an approximate model for the neighbouring core. Its effects are taken into account in the model spectra, but the description of the core is not accurate enough to include in the model optimisation. This would require a much more detailed analysis than possible here. In the procedure outlined in the next section, we therefore mask out those regions in the spectra strongly affected by the emission and absorption of the core.

\subsection{Optimising the fit}

Our model has four free parameters: the inclination $i$, the flatness parameter $f$, the stellar mass $M_{\star}$, and the angle of the velocity field $\alpha$. In addition, the abundances of the molecules are unknown. All other parameters are held fixed. Table 2 lists the parameters.

Considering the size of the parameter space and the time it takes to calculate a single spectrum ${ }^{1}$ the task of finding the parameter vector resulting in the best fit is non-trivial. This is further complicated by the degeneracy of the model results to different parameters. For example, increasing the abundance can have the same effect on the line intensity as increasing the inclination or the flatness, but these will have very different effects on the line profile shape.

Instead of calculating all possible models in the allowed parameter space, we use Voronoi tessellation of the parameter cube (see e.g. Kiang 1966, for details on Voronoi tessellation). A random set of $n$ points $p_{n}$ in the parameter cube is picked and model spectra are calculated for each of these. Then the parameter cube is divided into Voronoi cells, defined as the volume around a point $p_{i}$ in the parameter cube containing all points $q$ closer

\footnotetext{
${ }^{1}$ Depending on the species and the optical thickness, we can calculate a spectrum in between five minutes and half of an hour, on a stateof-the-art desktop processor.
}

to $p_{i}$ than to any other of the points $p_{n}(n \neq i)$. The parameter cube is scaled in arbitrary units, so that the allowed parameter ranges falls between 0 and 1 . On this dimensionless unit cube a simple metric in $d$ dimensions is used to define the cells,

$s^{2}=\sum_{i=1}^{d}\left(q_{i}-p_{i}\right)^{2}$

assuming that the solution depend linearly on all parameters. This assumption is not true especially for large values of $s$, but because we have no knowledge of the geometry of the parameter space, we use the simplest possible measure. In order to minimise the effect this has on our final solution we can increase the initial sample rate so that the average distance between the points becomes smaller. After one or two iterations, the volume of each cell is small enough so that the assumption of linear dependence is good. By scaling the parameters to the same range we make sure that each parameter is weighted equally in the distance measure.

The cell which contains the point $p_{i}$ resulting in the best fit is chosen, and a new set of random points are picked within this cell, and the procedure is iterated until sufficient convergence has been achieved. This method is only guaranteed to reach the true best fit if only one global minimum exist and if there are no (or few) local minima. To check whether we find the true optimum, we make several runs, with different randomly distribution initial points. We find that we always reach the same minimum, and conclude that local minima are few and not very deep.

For every calculated model spectrum, the fitness is evaluated by regriding the model spectrum to the channel width of the corresponding observed spectrum, centering it on the LSR velocity of $7.2 \mathrm{~km} \mathrm{~s}^{-1}$, and calculating the $\chi^{2}$ between the model and the observed spectrum,

$\chi^{2}=\frac{1}{M} \sum_{m} \frac{1}{N_{m}} \sum_{n} \frac{\left(I(n)_{\mathrm{obs}}-I(n)_{\mathrm{model}}\right)^{2}}{\sigma^{2}}$,

where $M$ is the number of spectra and $N$ is the number of velocity channels in the $m$ th spectrum. This way we give an equal weight to all spectra even though the number of channels vary in each spectrum. Those channels affected by the neighbouring core are not included in the $\chi^{2}$ measure. Every spectra has a fixed passband of $14 \mathrm{~km} \mathrm{~s}^{-1}$ so that an equal amount of baseline is included for each spectrum.

Using this method, with a set of 24 random points per iteration, we converge on an optimal solution after four to five iterations, corresponding to 10 to 12 days of CPU time. For practical reasons we initially chose only to consider the most structured lines $\left(\mathrm{CO}, \mathrm{HCO}^{+}\right.$and $\left.\mathrm{CS}\right)$, lowering the computational time to about a single day and getting a quick but rough handle on the initial parameter cube. We then included the other lines to obtain the overall best solution.

\subsection{Error estimates}

Getting a handle on the uncertainties in the obtained parameter values is a difficult matter due to the size and complexity of the parameter space. As mentioned above, we have no knowledge of the overall geometry of the parameter space and given the long computation time of the optimisation algorithm, we cannot make a correlation analysis of each pair of parameter and neither can we make $\chi^{2}$ surfaces. Still, it is very important to get an estimate on the stability and reliability of our solution. 

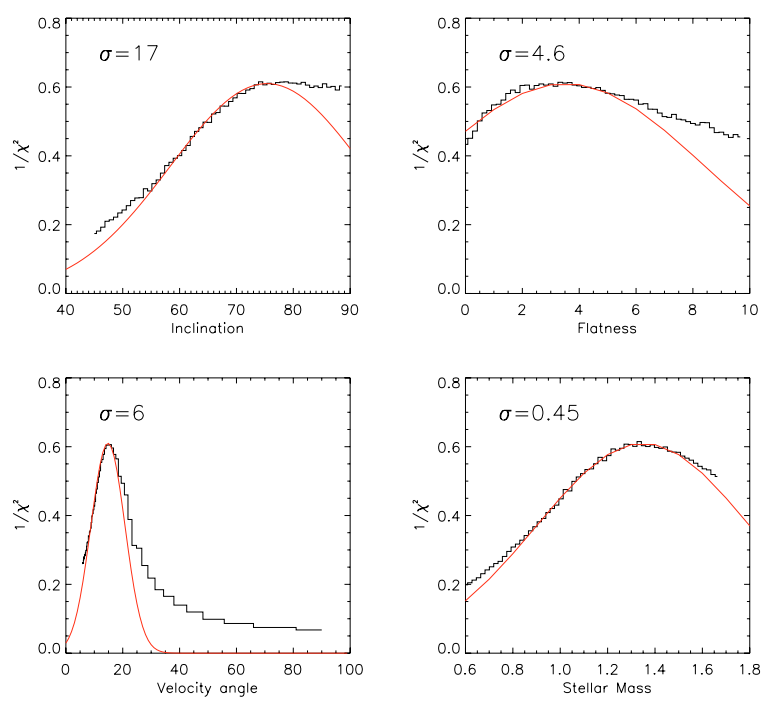

Fig. 4. The histograms show the $\chi^{2}$ distributions of models around the best fit position where only one parameter is varied at each time. A Gaussian, centered on the best fit in each panel, is fitted to the distributions. The dispersion of the Gaussians is given in each panel.

A simple error analysis is done for the four model dependent parameters, the flatness, the velocity angle, the stellar mass, and the inclination, by fixing three of the parameters at their best fit values, and calculating models in which the fourth parameter is gradually increased from its lower boundary to the upper boundary. Histograms of the resulting (inverse) $\chi^{2}$ values is shown in Fig. 4. The $\chi^{2}$ values are approximately normally distributed, with the main discrepancy in the high values of the inclination, velocity field, and the flatness. This relates to the non-linear nature of the trigonometric functions associated with these three parameters.

A Gaussian has been fitted to each of the histograms in Fig. 4. The centre of the Gaussian is fixed on the best fit value and the hight is fixed by the $\chi^{2}$ value of the best fit so that only the variance, $\sigma^{2}$, is free. Reasonable fits are achieved for each parameter with the $\sigma$ value given in each panel. These values are taken to be a rough estimate of the magnitude of the error in each of the four parameters. For the inclination and flatness, where the error is greater than the allowed parameter range, the error is of course determined by the physical constrains on the parameter value (e.g., the inclination cannot be greater than $90^{\circ}$ ). Note that the error bars are typically smaller than the explored range in each parameter by a factor of 2-10.

With this kind of one dimensional error analysis we do not take into account the fact that the parameters are likely to be highly correlated. A few exploratory calculations, where one parameter was held fixed at its best fit value while the other three where randomly pertubed around their best fit values, indicated that there indeed exist a strong correlation between the parameters. Indeed, a degeneracy exists between the central mass and the inclination, which again is degenerate with the flattening. Only a full parameter space study can fully disentangle this and is beyond the scope of this paper.

Because the abundance parameter mainly serves to scale the intensity in every channel of the spectrum, and does not change the shape of the profile much, this kind of error analysis is of little use. For any combination of the four free parameters that reproduces the observations, a corresponding abundance is found from the optically thin isotopic lines. These abundances are relatively insensitive to the exact geometry because of their optically thin nature. Therefore we assume that the error in the abundance values obtained here is entirely dominated by the $20 \%$ calibration error of the observed spectra.

Throughout this work we have assumed constant abundance for all the molecular species. In reality, abundances will depend on the chemistry and molecules will freeze out below a certain temperature. This gives rise to a drop in the abundances at a certain radius and it will affect, to some extent, the shape of the profiles but more prominently, the line ratios. Specifically, by removing low temperature material from the gas phase, low excitation lines become relatively weaker. Our model does not suffer from the problem of over-producing the low $J$ lines, except for the case of $\mathrm{HCO}^{+}$; a more complex abundance model would likely provide a better fit to the $J=1-0$ and 3-2 lines. However, this would require a careful chemical analysis which is beyond the scope of this paper. A few tests showed that letting $\mathrm{CO}$ freeze out at $20 \mathrm{~K}$ does not change the best fit parameters significantly, except for the abundance which will then have to be re-optimised.

\section{Results}

Figure 1 compares the data to the synthetic spectra based on the best fit model obtained with the optimisation procedure described above. The results for the combined emission of L1489 IRS and the neighbour core is shown in Fig. 5. Because the emission from the core only contributes to the low $J$ lines, this figure only shows the species in which the combined spectrum show any difference from the L1489 IRS spectrum alone. For all species not shown in Fig. 5, the combined spectra is indistinguishable from the one shown in Fig. 1. Table 2 lists the parameters of the best fit.

The inclination angle of $74^{\circ}$ falls within the range of $60^{\circ}$ to $90^{\circ}$ which is inferred from the scattered light image of Padgett et al. (1999) and the modelling of the infrared spectral energy distribution Kenyon et al. (1993b). Section 3.2.2 shows that this inclination and the flattening parameter $f=3.8$ reproduce the scattered light image, including the detectability of the central star. The resulting density distribution can be well approximated by a disk with a vertical density distribution $\propto \mathrm{e}^{-z^{2} / 2 h^{2}}$ and a scale height of $h \approx 0.57 R$. The maximum deviation of this approximation is only $4 \%$, up to an angle of $60^{\circ}$ above the midplane. Hogerheijde (2001) described L1489 IRS with flared disc with an adopted density scale height of $h=0.5 R$, so our best fit model shows that the structure might in fact be flatter than previously assumed. However, Hogerheijde (2001) used an inclination of $90^{\circ}$ for the flared disc (a flat disc at $i=60^{\circ}$ was also tried), so the projected column density distribution are quite similar in both cases.

The best fitting velocity vector makes an angle of only $15^{\circ}$ with respect to the azimuthal direction, consistent with Hogerheijde (2001) who shows that rotation is the primary component in the velocity field. However, our central stellar mass of $1.35 M_{\odot}$ is considerably higher than the $0.65 M_{\odot}$ derived by Hogerheijde. Part of this is due to different definitions of the velocity field. Hogerheijde reports a mass expected for Keplerian motion based on the azimuthal component of the velocity field alone, while the mass derived here results in $\cos \alpha$ times the Keplerian velocity (Eq. (4)). In addition, a different inclination is found. These two factors together would give a mass of $1.2 \mathrm{M}_{\odot}$ for our model. This mass is still $80 \%$ higher than that from Hogerheijde but our best fit has a $\chi^{2}$ value of 1.69 . That is nearly half that of the best model of Hogerheijde of $\sim 3$. We ascribe this difference to a more thorough search of the parameter space. 


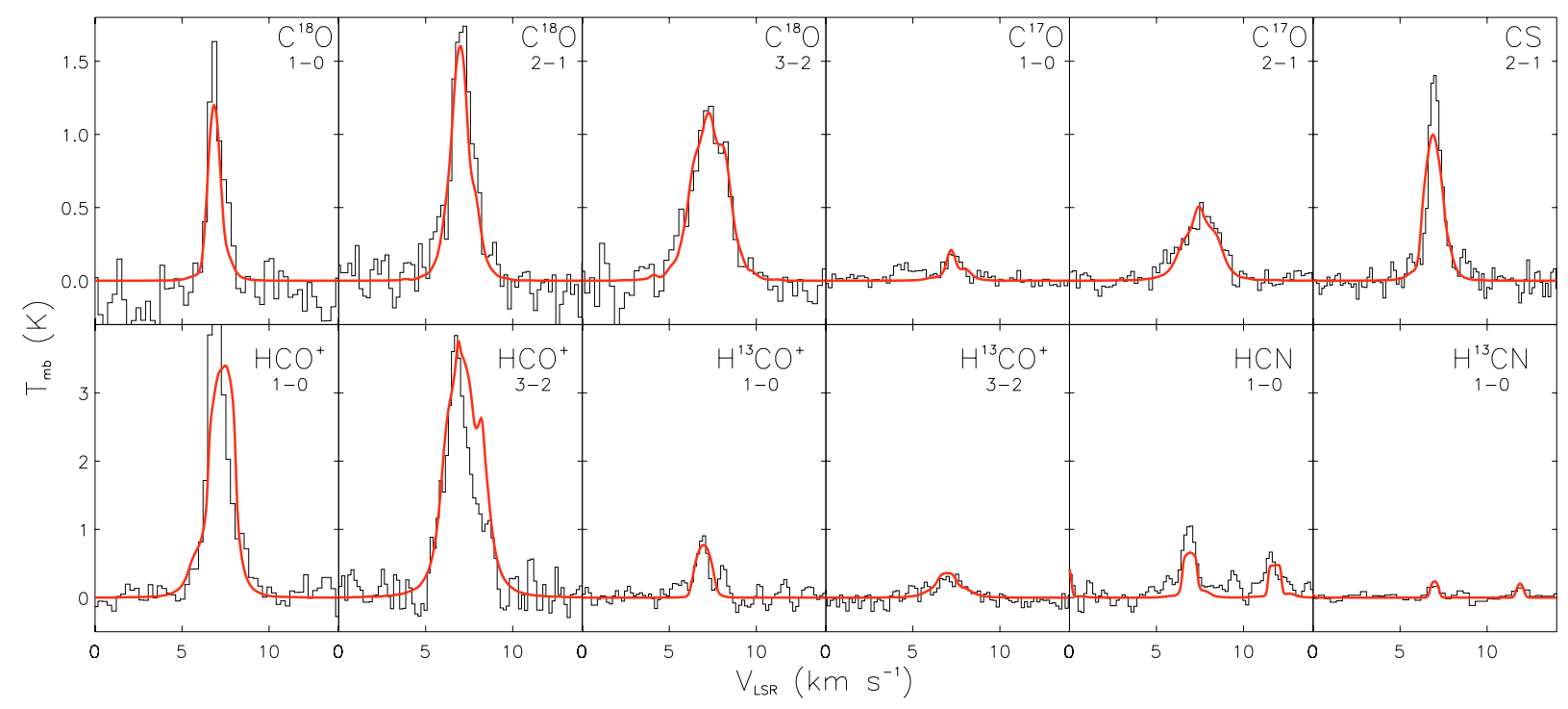

Fig. 5. The combined emission from the L1489 IRS model and the neighbour cloud core. Only spectra which are affected significantly by the cloud core emission are shown here.

Table 2. Best fit parameters.

\begin{tabular}{lc}
\hline \hline Free parameter & Value \\
\hline Inclination of disc, $i$ & $74^{\circ}$ \\
Flatness of disc, $\sin ^{f}(\theta)$ & 3.8 \\
Central mass, $M_{*}$ & $1.35 M_{\odot}$ \\
Velocity angle, $\alpha$ & $15^{\circ}$ \\
& \\
Abundance of $\mathrm{CO}^{*}$ & $3.5 \times 10^{-5}$ \\
Abundance of CS & $0.5 \times 10^{-9}$ \\
Abundance of SO & $2.0 \times 10^{-9}$ \\
Abundance of $\mathrm{HCO}^{+}$ & $1.9 \times 10^{-9}$ \\
Abundance of $\mathrm{HCN}$ & $0.2 \times 10^{-9}$ \\
Abundance of HNC & $0.2 \times 10^{-9}$ \\
Abundance of CN & $0.2 \times 10^{-9}$ \\
Abundance of $\mathrm{H}_{2} \mathrm{CO}$ & $0.7 \times 10^{-9}$ \\
\hline Fixed parameters & \\
\hline Disc mass & $0.097 M_{\odot}$ \\
Temperature slope & -0.35 \\
Temperature at $1000 \mathrm{AU}$ & $19.42 \mathrm{~K}$ \\
Density slope & -1.8 \\
Turbulent velocity, $F W H M$ & $0.2 \mathrm{~km} \mathrm{~s} \mathrm{~s}^{-1}$ \\
Outer radius & $2000 \mathrm{AU}$ \\
Distance & $140 \mathrm{pc}$ \\
\hline
\end{tabular}

* The main isotopic abundance has been derived from the $\mathrm{C}^{18} \mathrm{O}$ abundance using a ${ }^{16} \mathrm{O} /{ }^{18} \mathrm{O}$ ratio of 540 (Wilson \& Rood 1994).

The best fitting abundances are consistent with the abundances obtained for L1489 IRS in (Jørgensen et al. 2004) to within a factor of 2-3 although the values obtained here are all higher. This may be due to higher line opacities in our model as a result of the different adopted velocity fields; Jørgensen et al. do not include a systematic velocity field and only a single turbulent line width. Consistent with this previous work, we find no evidence for depletion of $\mathrm{CO}$, in accord with the relatively high temperatures exceeding the $20 \mathrm{~K}$ evaporation temperature of $\mathrm{CO}$ throughout most of the disc. Another interesting finding is the $\mathrm{CN} / \mathrm{HCN}$ abundance ratio of 1.0 , which is more reminiscent of dark clouds than of circumstellar discs, suggesting that chemically, L1489 IRS is close to its original cloud core and that photo-dissociation does not play a major role yet (Thi et al. 2004).

\subsection{Quality of the best fit}

The overall correspondence of our model to the data is good and most of the spectra are well reproduced. The line widths of $\mathrm{C}^{18} \mathrm{O}$ and $\mathrm{C}^{17} \mathrm{O}$ are well reproduced, and the $\mathrm{C}^{17} \mathrm{O} 2-1$ and 3-2 lines are found to exclusively trace L1489 IRS and be uncontaminated by the neighbouring core. All three $\mathrm{C}^{18} \mathrm{O}$ and the $\mathrm{C}^{17} \mathrm{O} 1-0$ line have narrow line peaks that originate in the neighbour core; some $\mathrm{C}^{18} \mathrm{O} J=1-0$ emission that is not reproduced can either be caused by additional material along the line of sight, or be due to the approximate nature of our description of the core.

The sulfur bearing lines are not very intense and show little structure. Again we see a narrow peak in the CS $J=2-1$ almost entirely accounted for by the neighbouring core and perhaps also in the SO line. The non-detection of $\mathrm{C}^{34} \mathrm{~S} 2-1$ places an upper limit on the CS abundance in the neighbouring cloud of $2 \times 10^{-9}$. The CS 7-6 line is poorly fit, but the observed spectrum has low signal-to-noise.

The $\mathrm{HCO}^{+}$lines are the strongest among our sample and show most structure in their profiles. It was important to be able to reproduce the double peak in the $\mathrm{HCO}^{+} J=4-3$ line because this feature is a very clear tracer of the velocity structure in L1489 IRS. Our model is able to reproduce this feature. However, we do not observe the double peak in the $\mathrm{HCO}^{+} J=$ 3-2 line but only a slight asymmetry. This provided a major constraint on the velocity field. The neighbour core does not contribute in the 4-3 line but it makes up for almost all of the excess emission seen in the 1-0 and 3-2 lines of $\mathrm{HCO}^{+}$.

Of the nitrogen bearing species, $\mathrm{HCN} J=1-0$ with its three hyperfine components shows very narrow lines, which is well reproduced by the neighbour core model which dominates the emission. The HCN $J=4-3$ line is much broader and uncontaminated by the neighbouring core. A narrow peak in the spectrum at $8 \mathrm{~km} \mathrm{~s}^{-1}$ cannot be due to the neighbour core and we assume it is noise. The HNC 4-3 line has a rather low signal to noise ratio and can only provide a reliable estimate within a factor of a few. $\mathrm{CN} J=1-0$ also has a low signal to noise ratio, 


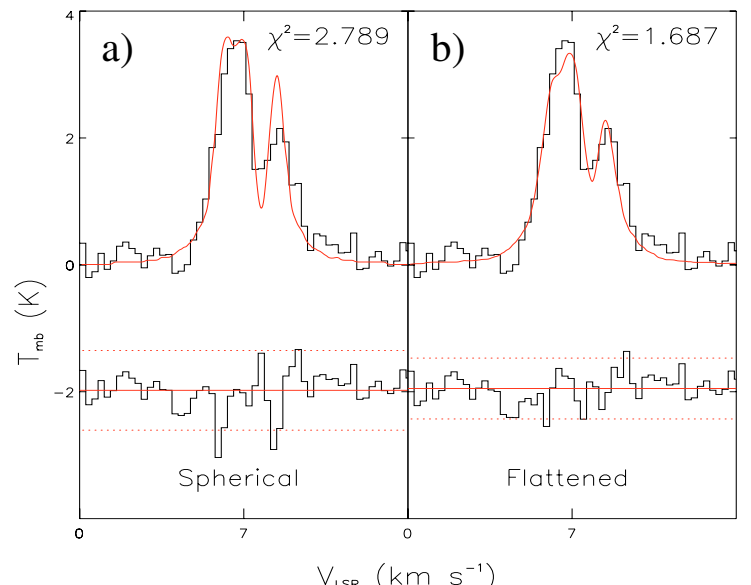

Fig. 6. a) Best possible fit with a spherical model to the $\mathrm{HCO}^{+} J=$ 4-3 line. b) The best fit to the same line where the flattening $f$ and the inclination $i$ are kept as free parameters. Below, the residuals are shown with the mean and two standard deviations indicated.

but in the case of $\mathrm{CN}$, the abundance is well constrained by the $J=3-2$ line.

All spectra discussed above have been obtained with singledish telescopes that do not resolve the 2000 AU radius source. One can wonder if it is justifiable to use a non-spherical model when the single-dish observations does not contain any spatial information. Although the scattered-light and interferometer images show that the structure is non-spherical, one could argue that introducing the flattening is simply a way to improve the fit by adding a free parameter. However, this is not the case. Figure 6 shows the best fit to the $\mathrm{HCO}^{+} J=4-3$ line, with a spherical model $(f=0)$ and a model where the flattening and inclination are free parameters. The flattened model provides a considerably better fit with lower $\chi^{2}$ value. And, more importantly, the spherical model shows too much self absorption. Our optimisation algorithm returns the model which minimises the difference between data and model for each velocity channel. In a spherical model the column simply becomes too high. By flattening the model and adjusting the inclination, we can exactly reproduce the right amount of self absorption seen in the data while keeping the total column density high enough to produce the right line strength. The degeneracy between these two parameters are resolved by the velocity field, and thus it turns out that it is actually possible to retrieve spatial information from single dish observations.

Ward-Thompson \& Buckley (2001) have argued that the amount of self-absorption can also be regulated by adjusting the turbulent velocity dispersion. We can indeed change the quality of the spherical fit by changing the amount of turbulence. We cannot, however, do that without also changing the velocity distance between the two peaks. Thus in order to fit the line width we must decrease the mass and thereby the magnitude of the velocity field, which no longer reproduces the observed infall asymmetry. We therefore find that varying the turbulent velocity width in a spherical model does not reproduce the observations.

\subsection{Comparison to other observations}

With the fit parameters derived above, we can now test our model by comparison to other observations of L1489 IRS not used in the fit. The neighbouring cloud is not considered in the following.
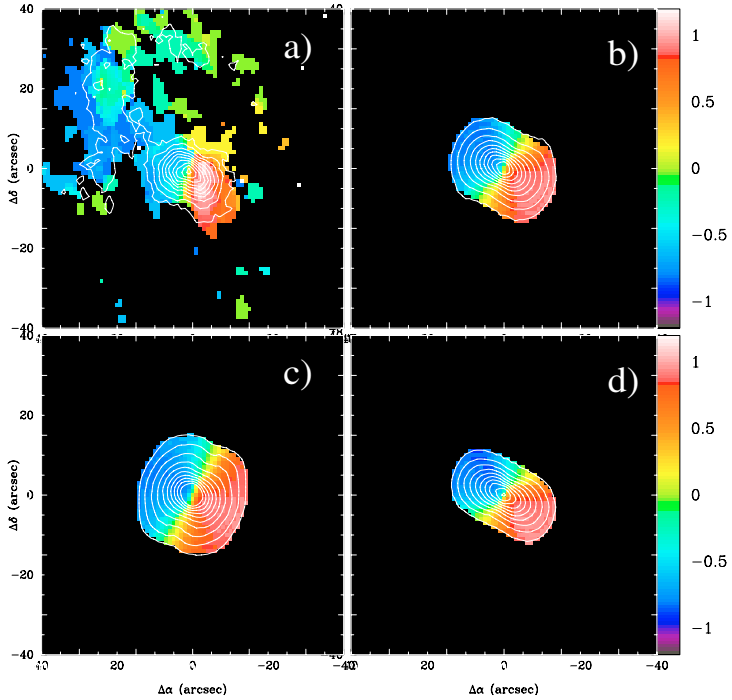

Fig. 7. a) Interferometer map in $\mathrm{HCO}^{+} J=1-0$; b) the corresponding synthesised map based on our best model; c) and d) models with $f=1$ and $f=8$ respectively. The white contour lines show the integrated intensity, starting at $0.25 \mathrm{Jy} \mathrm{bm}^{-1}$ and increasing in steps of $0.5 \mathrm{Jy} \mathrm{bm}^{-1}$. The colour scale shows the velocity centroid in units of $\mathrm{km} \mathrm{s}^{-1}$.

\subsubsection{Interferometer image of $\mathrm{HCO}^{+} 1-0$}

We have used our model to produce a synthetic interferometer map of the $\mathrm{HCO}^{+} J=1-0$ emission which can be directly compared to the data presented by Hogerheijde (2001). Figure 7 compares the model predictions of the integrated intensity and velocity centroid maps to the observations reproduced from Hogerheijde (2001). We also show a model with $f=1$ and on with $f=8$ for comparison. The model images were made by taking the unconvolved image cubes from RATRAN and then, using the $(u, v)$ settings from the original data set, making synthetic visibilities with the "uvmodel" task from the MIRIAD software package. This is what we would get if the interferometer observed our model object. Then we applied the usual deconvolution with the invert, clean, and restore routines in order to reconstruct an image from the visibilities.

The resulting synthetic image of our best fit model resembles the observations closely within the uncertainty of the abundance which sensitively affects the apparent size, when it is taken into account that the observations also partially recover the neighbouring core that is ignored in the model (Fig. 7, panel b). However, the $f=8$ model in panel d) is also in good agreement with the data which partially can be explained by the fact that, due to the non-linearity of the sine function, the difference between an $f=3$ and $f=10$ model is much less than the difference between an $f=1$ and $f=3$ model. This is also reflected in Fig. 2. In any case, panel c) is obviously in poor agreement with the data, which shows that in order to fit the interferometer data, a flattened structure is needed.

This kind of analysis is very useful to investigate the spatial distribution of the emission which is lacking in the single dish data. Importantly, we see that the flattening which we introduced and the amount of which we determined from the line profile results in a projected shape that is very close to what we see in the data image. Also, because the cuts are the same in both panels, the extent of the emission and therefore the physical size scale of the model is consistent. 

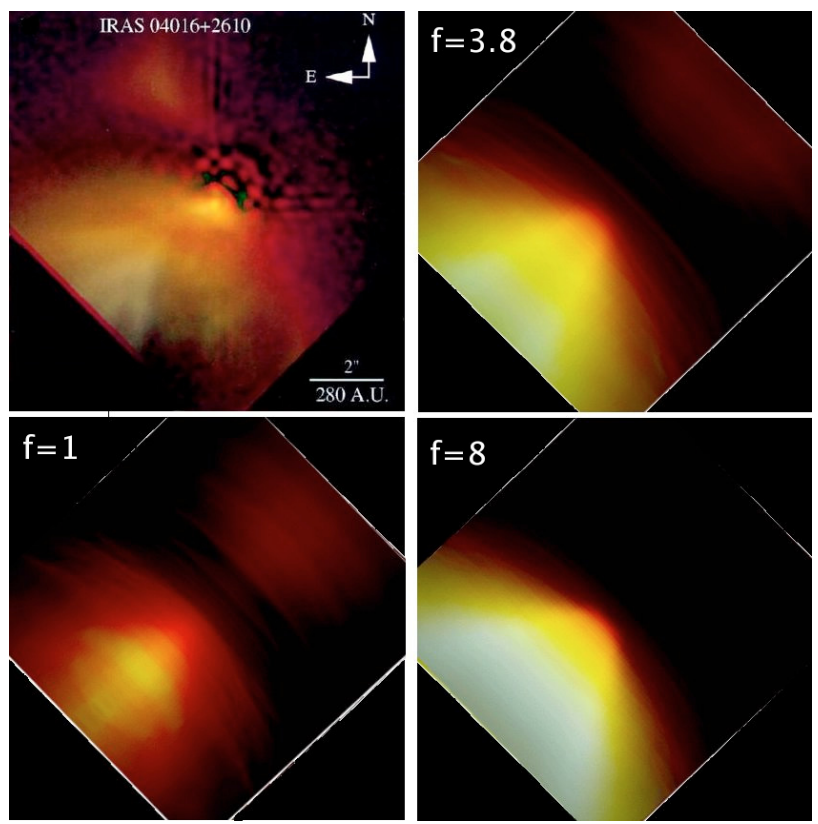

Fig. 8. The top left panel shows the false colour NICMOS image of L1489 IRS from Padgett et al. (1999). In the three remaining panels are shown synthetic three-colour composite images based on our model.

\subsubsection{Near-infrared image}

We subsequently test our best-fit model through a comparison with the near-infrared scattered light image of Padgett et al. (1999). A reproduction of this image is shown in the top left panel of Fig. 8. Using the density structure and inclination found in Sect. 3 and the properties of the central star as described in the introduction as input, we calculated the scattering light emission with RADMC, a two-dimensional radiation transfer code (Dullemond \& Dominik 2004). Then, using the ray-tracing code RADICAL (Dullemond \& Turolla 2000), we extract the fluxes over the full spectral range of 1 to $850 \mu \mathrm{m}$ and image our model in the $F 110 W, F 160 W, F 210 W$ NICMOS bands.

Our best fit model of Sect. 3 automatically provides a good fit to the sub-millimetre part of the spectral energy distribution, confirming that our model is consistent with the results of Jørgensen et al. (2002). The near-infrared observations are more difficult to match. The column density in the inner part is too high to provide the clear view of the central star that shows prominently in NICMOS images. However, if we reduce the scale height in the inner $250 \mathrm{AU}$ to $h=0.15 R$, the column density is reduced and the central star becomes detectable in the near-infrared. This adaptation does not affect the sub-millimetre emission or the molecular line intensities viewed in the much larger single-dish beams. Recently obtained high-resolution data from the Submillimeter Array (SMA) in Hawaii may be able to test this assumption of the geometry in the inner $2^{\prime \prime}=280 \mathrm{AU}$ (Brinch et al., in prep.).

The resulting three-colour composite images of our best fit model as well as a $f=1$ and $f=8$ model, is shown in Fig. 8 . Although this can only be a qualitative comparison, our model is able to reproduce most of the striking features evidenced by the observations. The opening angle of the dust cavity is found to be somewhat dependent on the flattening parameter $f$ in our model. Although it is difficult to judge the agreement, this result seems to favour a relatively low value of $f$ as opposed to what we found above for the interferometric map. The non-monotonic behaviour along the axis in the $f=1$ model is a combination of

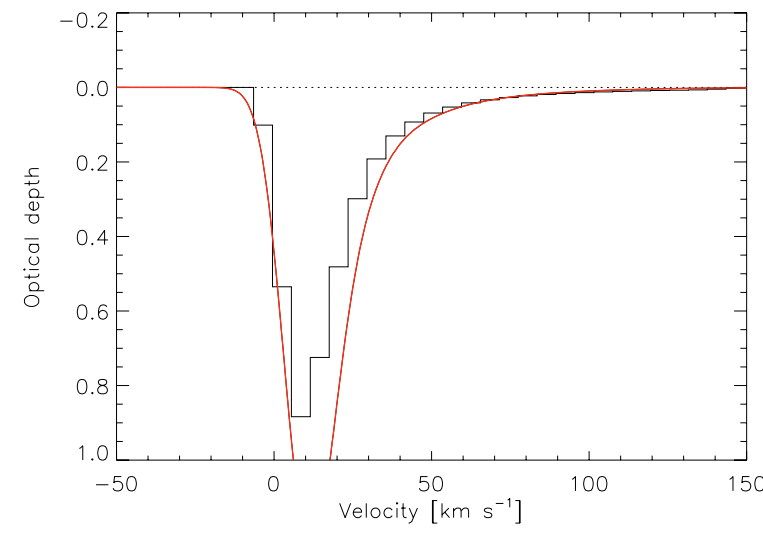

Fig. 9. An average of the observed ${ }^{12} \mathrm{CO} \mathrm{P}(6)-\mathrm{P}(15)$ lines with a similarly averaged model result over plotted.

the finite gridding of the density structure and the effect of scattering. It has a very narrow cavity and so the base of the scattering nebula is actually absorbed by the high density material in the inner part. In the other models the cavity is large enough to allow all the scattered photons to escape.

We also reproduce the near-infrared colours, confirming our adopted density distribution between $\sim 100$ AU and 2000 AU. Each of the NICMOS fluxes are reproduces to within about $40 \%$. Although a detailed modelling of L1489 IRS on these scales where most of the near-infrared emission is coming from was beyond the scope of this paper, we present this prediction to demonstrate that it is possible to combine the information contained in near-infrared images and sub-millimetre single dish measurements to obtain a self-consistent model on scales ranging from within a few AU up to several thousand AU.

The cavity seen in the NICMOS image is likely associated with a molecular outflow. However, Hogerheijde et al. (1998) show that L1489 IRS only drives a modest ${ }^{12} \mathrm{CO}$ outflow, and little or no impact on the line profiles is expected. Therefore, an outflow has not been incorporated into the model described in this paper.

\subsection{3. ${ }^{12} \mathrm{CO} 4.7 \mu \mathrm{m}$ absorption bands}

Finally we apply our model to fit the CO ro-vibrational absorption bands, again by using RATRAN. Here we assume that initially all $\mathrm{CO}$ molecules are in the vibrational ground state $(v=0)$ but can be excited to a $v=1$ state by absorption of a photon from the central star, producing the absorption lines in the $P$ and $R$ branches corresponding to $\Delta J= \pm 1$ transitions. Observations of these bands from the Keck/NIRSPEC instrument has been presented by Boogert et al. (2002). The spectrally resolved absorption lines revealed inward motions up to $100 \mathrm{~km} \mathrm{~s}^{-1}$. Using the same method as Boogert et al. we calculated the ro-vibrational absorption lines in our model, and plot the average of the $P(6)-P(15)$ lines in Fig. 9.

We find that our model fits the data as well as the results from Boogert et al., who use a contracting flared disc with power laws for the temperature, density, and infall velocity. This means that the amount of inward velocity that we obtained from the fit to the single dish lines together with the adopted density profile can explain the observed infall, even at radii much smaller than probed with the single dish lines. In Fig. 9 we see that absorption is present all the way up to at least $100 \mathrm{~km} \mathrm{~s}^{-1}$. In our model, this velocity translates into a radius of $0.02 \mathrm{AU}$. Material absorbing at 50 and $20 \mathrm{~km} \mathrm{~s}^{-1}$ is located at 0.06 and 0.4 AU respectively. 


\section{Discussion}

In this paper we derive an accurate model for the circumstellar material of L1489 IRS. We find that it is well described by a flattened structure with a radius of $2000 \mathrm{AU}$, in sub-Keplerian motion around a $1.35 M_{\odot}$ central star. While the structure resembles discs found around T Tauri stars (e.g. Simon et al. 2000), its $2000 \mathrm{AU}$ radius is much larger than T Tauri discs (typically several hundred AU). Also, discs around T Tauri stars are often well described by pure Keplerian motions except for a few cases, e.g. AB Aur, in which outwards non-Keplerian motions are measured (Piétu et al. 2005; Lin et al. 2006). In this section we discuss the evolutionary state of L1489 IRS, and particularly whether it represents a unique case or if other forming stars may go through a similar stage. We start by deriving the life span of the current configuration. We then explore the relation of L1489 IRS to its neighbouring core. Finally, we discuss a number of open questions that only new observations can answer.

By integrating the trajectory of a particle at $2000 \mathrm{AU}$ we find the infall time scale to be $2.3 \times 10^{4}$ years. Dividing the $0.097 M_{\odot}$ of the circumstellar material yields a mass accretion rate of $4.3 \times$ $10^{-6} M_{\odot} \mathrm{yr}^{-1}$. Estimating the radius of the central star from the mass-radius relation (Stahler 1988) to be $4 R_{\odot}$ results on an accretion luminosity of $L_{\mathrm{acc}}=\mathrm{G} M_{*} \dot{M} / R_{*} \approx 46 L_{\odot}$ corresponding to about ten times the observed bolometric luminosity from the YSO Kenyon et al. (1993a). This suggest that not all inspiraling material falls directly onto the star. The result is somewhat higher than the one found by Hogerheijde (2001), although the modelling approach used in that paper was completely different.

In our model we also assume a constant angle $\alpha$ for the direction of the velocity vector. In reality, this direction could vary with radius as the angular momentum distribution changes. For example, at smaller radii, $\alpha$ could be smaller as the velocity field more closely resembles pure Keplerian rotation. The inspiral time, and mass accretion rate, can therefore be very different. Only higher resolution observations can investigate this further.

Is L1489 IRS in any way special? No objects like it are reported in the literature. Since its life time is of the order of a few $10^{4} \mathrm{yr}$, roughly $5-10 \%$ of the embedded phase, more objects like it would be expected. It is possible that L1489 IRS was formed out of a core with unusually large angular momentum, which led to the formation of an untypically large disc. We cannot exclude that possibility, but hydrodynamical simulations are required to investigate how much angular momentum would be required, and if turbulent cloud cores can contain such amounts of rotation.

An intriguing possibility is that the proximity of the neighbouring core is in some way related to L1489 IRS' special nature. The core is $60^{\prime \prime}$ (8400 AU) away in projection, and located in front of L1489 IRS, likely by a distance of comparable magnitude. Its systemic velocity is $0.4 \mathrm{~km} \mathrm{~s}^{-1}$ lower than that of L1489 IRS itself, indicating that L1489 IRS and the core currently are moving away from one another, at least in the direction along the line of sight. Could the neighbouring core be feeding material onto the disc of 11489 IRS? The velocity gradient is such that it merges smoothly with the velocities in the core (see Fig. 7). This suggest that a physical link between the core and the disc may exist. If the core feeds material onto the disc, this could be significant source of angular momentum, thus keeping the disc large. It is, however, not easy to understand why gas in the core would be gravitationally bound to the L1489 IRS star, because of the significant mass reservoir in the core itself of $2.9 M_{\odot}$.
Another hypothesis is that L1489 IRS actually originated inside the core, but has since migrated away. Its current velocity offset of $0.4 \mathrm{~km} \mathrm{~s}^{-1}$ is sufficient to move it to its current location $60^{\prime \prime}$ away in a few times $10^{5} \mathrm{yr}$, the typical life time of an embedded YSO. We of course do not know how far its offset along the line of sight is, or what its three-dimensional velocity vector is like. Its line-of-sight velocity of $0.4 \mathrm{~km} \mathrm{~s}^{-1}$ is not very different from the velocity dispersion of $\mathrm{T}$ Tauri stars and the turbulent motions in cloud complexes. One could propose that L1489 IRS' natal core was a turbulent, transient structure, and that, once formed, the YSO migrated with its gravitationally bound disc-like environment to a location outside the surrounding cloud core, in effect "stripping" the Class I object from most of its envelope. In this scenario we would now be seeing the inner, rotating Class I envelope around L1489 IRS unobscured by the outer envelope. This might account for the different appearance of L1489 IRS.

This hypothesis can be tested in two ways. First, "normal" Class I objects can be studied at high spatial resolution and in dense gas tracers to explore if the inner envelope is dominated by rotation on 1000 AU scales. Second, hydrodynamical calculations can be used to explore on what time scales newly formed YSOs can migrate away from their nascent cloud core. Searching for other objects like L1489 IRS would also be very useful. Potential targets would appear compact in sub-millimetre continuum images, but show strong emission lines in dense gas tracers. L1489 IRS shows $\mathrm{HCO}^{+}$lines with intensities of several $\mathrm{K}$, while a few tenths of $\mathrm{K}$ is more typical for $\mathrm{T}$ Tauri discs (Thi et al. 2004).

\section{Summary}

We have made a two-dimensional axi-symmetric disc-like model of the Young Stellar Object L1489 IRS. Line radiation transfer calculations produce synthetic spectra which can be directly compared to observations. We show that a flattened model gives a better description than a spherical one. Our model also reproduces millimeter interferometric imaging, nearinfrared scattered light images, and CO ro-vibrational absorption spectroscopy.

We conclude further that the central star has a mass of $1.35 M_{\odot}$. The velocity vectors make an angle of $15^{\circ}$ with the azimuthal direction. The velocity field is dominated by rotation, while small but significant amount of infall are present.

A neighouring cloud core is present next to L1489 IRS. This cloud is well modelled by generic dark cloud parameters and we argue that it is likely situated in front of L1489 IRS. We speculate that this core could be feeding high angular-momentum material onto the L1489 IRS disc, explaining the unusual size of this object. Another explanation could be that L1489 IRS is an ordinary Class I object which has migrated away from its parental core, leaving it surrounded by only its gravitationally bound inner envelope. If this is true, L1489 IRS may provide valuable insight on the formation of protoplanetary discs.

This paper shows that it is possible to construct a global model of a Young Stellar Object that is able to fit observations on a wide range of spatial scales. Single dish line observations provide enough information to make highly detailed models of circumstellar structures, even on scales that are unresolved. However, on scales as small as $100 \mathrm{AU}$, the description may no longer be accurate, as evidenced by the near-infrared scattered light which suggest additional flattening of the disclike structure. Recently obtained interferometer imaging with the 
Submillimeter Array may provide more insight on the inner several hundred AU around L1489 IRS (Brinch et al., in prep.).

Acknowledgements. This research was supported by the European Research Training Network "The Origin of Planetary Systems" (PLANETS, contract number HPRN-CT-2002-00308). C.B. is supported by the European Commission through the FP6 - Marie Curie Early Stage Researcher Training programme. The research of MRH is supported by a VIDI grant from the Nederlandse Organisatie voor Wetenschappelijk Onderzoek. The research of J.K.J. is supported by NASA Origins Grant NAG5-13050

\section{References}

Adams, F. C., Shu, F. H., \& Lada, C. J. 1988, ApJ, 326, 865

André, P., Ward-Thompson, D., \& Motte, F. 1996, A\&A, 314, 625

André, P., Ward-Thompson, D., \& Barsony, M. 2000, Protostars and Planets IV, 59

Boogert, A. C. A., Hogerheijde, M. R., \& Blake, G. A. 2002, ApJ, 568, 761

Dullemond, C. P., \& Dominik, C. 2004, A\&A, 417, 159

Dullemond, C. P., \& Turolla, R. 2000, A\&A, 360, 1187

Gregersen, E. M., \& Evans, N. J. 2000, ApJ, 538, 260

Guilloteau, S., \& Dutrey, A. 1998, A\&A, 339, 467

Hogerheijde, M. R. 2001, ApJ, 553, 618

Hogerheijde, M. R., \& Sandell, G. 2000, ApJ, 534, 880

Hogerheijde, M. R., \& van der Tak, F. F. S. 2000, A\&A, 362, 697

Hogerheijde, M. R., van Dishoeck, E. F., Blake, G. A., \& van Langevelde, H. J. 1997, ApJ, 489, 293
Hogerheijde, M. R., van Dishoeck, E. F., Blake, G. A., \& van Langevelde, H. J. 1998, ApJ, 502, 315

Jørgensen, J. K., Schöier, F. L., \& van Dishoeck, E. F. 2002, A\&A, 389, 908 Jørgensen, J. K., Schöier, F. L., \& van Dishoeck, E. F. 2004, A\&A, 416, 603 Kenyon, S. J., Calvet, N., \& Hartmann, L. 1993a, ApJ, 414, 676

Kenyon, S. J., Whitney, B. A., Gomez, M., \& Hartmann, L. 1993b, ApJ, 414, 773

Kiang, T. 1966, Z. Astrophys., 64, 433

Koerner, D. W., \& Sargent, A. I. 1995, AJ, 109, 2138

Lada, C. J., \& Wilking, B. A. 1984, ApJ, 287, 610

Lin, S.-Y., Ohashi, N., Lim, J., et al. 2006, ApJ, 645, 1297

Lizano, S., \& Shu, F. H. 1989, ApJ, 342, 834

Mardones, D., Myers, P. C., Tafalla, M., et al. 1997, ApJ, 489, 719

Myers, P. C., Fuller, G. A., Mathieu, R. D., et al. 1987, ApJ, 319, 340

Nenkova, M., Ivezic, Z., \& Elitzur, M. 1999, LPI Contributions, 969, 20

Ossenkopf, V., \& Henning, T. 1994, A\&A, 291, 943

Padgett, D. L., Brandner, W., Stapelfeldt, K. R., et al. 1999, AJ, 117, 1490

Piétu, V., Guilloteau, S., \& Dutrey, A. 2005, A\&A, 443, 945

Reipurth, B., Jewitt, D., \& Keil, K., ed. 2006, in press, Protostars and Planets V (University of Arizona Press)

Schöier, F. L., van der Tak, F. F. S., van Dishoeck, E. F., \& Black, J. H. 2005, A\&A, 432, 369

Shu, F. H. 1977, ApJ, 214, 488

Simon, M., Dutrey, A., \& Guilloteau, S. 2000, ApJ, 545, 1034

Stahler, S. W. 1988, ApJ, 332, 804

Stamatellos, D., Whitworth, A. P., André, P., \& Ward-Thompson, D. 2004, A\&A, 420, 1009

Thi, W.-F., van Zadelhoff, G.-J., \& van Dishoeck, E. F. 2004, A\&A, 425, 955

Ward-Thompson, D., \& Buckley, H. D. 2001, MNRAS, 327, 955

Wilson, T. L., \& Rood, R. 1994, ARA\&A, 32, 191 